\title{
Eddy-Current Distortion Correction and Robust Tensor Estimation for MR Diffusion Imaging
}

\author{
J.-F. Mangin ${ }^{1,3}$, C. Poupon ${ }^{1,2,3,4}$, C. Clark ${ }^{1,3}$, D. Le Bihan ${ }^{1,3}$, and I. Bloch ${ }^{2,3}$ \\ 1 Service Hospitalier Frédéric Joliot, CEA, 91401 Orsay, France \\ mangin@shfj.cea.fr, http://www-dsv.cea.fr/ \\ 2 Département Signal et Images, CNRS URA 820, ENST, Paris \\ ${ }^{3}$ Institut Fédératif de Recherche 49 \\ ${ }^{4}$ GE Medical Systems, Buc, France
}

\begin{abstract}
This paper presents a new procedure to estimate the diffusion tensor from a sequence of diffusion-weighted images. The first step of this procedure consists of the correction of the distortions usually induced by eddy-current related to the large diffusion-sensitizing gradients. This correction algorithm relies on the maximization of mutual information to estimate the three parameters of a geometric distortion model inferred from the acquisition principle. The second step of the procedure amounts to replacing the standard least squares based approach by the Geman-McLure M-estimator, in order to get rid of outlier related artefacts. Several experiments prove that the whole procedure highly improves the quality of the final diffusion maps.
\end{abstract}

\section{Introduction}

There is currently considerable interest in the use of MRI for imaging the apparent diffusion of water in brain tissues [13. When anisotropy of the 3D diffusion process is of interest, for instance for fiber bundle tracking [18], a symmetric diffusion tensor $D$ has to be calculated for each voxel from a series of diffusionweighted volumes [3, 2]. Each such volume is acquired with a different applied diffusion-sensitizing gradient [21]. These gradients are applied in order to vary a symmetric matrix $b\left(\mathrm{~s} / \mathrm{mm}^{2}\right)$ that depends on the gradient direction, strength and timing [15. The diffusion-sensitizing gradient affects the signal intensity of any given voxel in a manner that can be described by the linear equation:

$$
\ln S(\boldsymbol{b})=\ln S(0)-D_{x x} b_{x x}-2 D_{x y} b_{x y}-2 D_{x z} b_{x z}-D_{y y} b_{y y}-2 D_{y z} b_{y z}-D_{z z} b_{z z},
$$

where $S$ denotes the signal of the selected voxel. When a sufficient number of different $b$ matrices is used, the diffusion tensor $D$ can be estimated.

Such calculations are simple if each voxel in the different volumes represents the same point in the anatomy of the subject, but can be impractical if different volumes of the series are distorted relative to each other. Diffusion-weighted images, however, are often acquired using Echo-Planar Imaging (EPI), to reduce acquisition time. Unfortunatelly, this fast acquisition scheme is highly sensitive 
to eddy currents induced by the large diffusion gradients [8]. These eddy currents can cause significant distortions in the phase-encoding direction where the image bandwidth is quite low (see Fig. 1). Since the degree and nature of this artefact typically vary both with the strength and orientation of the diffusion-sensitizing gradient, distortions can dramatically change the direction of highest diffusion supposed to correspond to fiber direction.

The methods for reducing the effects of eddy currents may be divided into four categories. The first one simply consists of modifications of the gradient sequences [1]. This approach, however, seems unsufficient to get completely rid of artefacts. Other approaches are retrospective and can be considered as registration methods. Some of them which rely on MR physics require additional experimental data 11]. Others simply use a distortion geometric model inferred from the acquisition principle, which leads to estimate a few parameters using a standard similarity measure like cross-correlation [8, 6, 4]. The last kind of approaches stem from recent progress in the definition of robust similarity measures. To our knowledge, such approaches have only been applied in functional MRI to correct for distortions induced by susceptibility artefacts in EPI, using a free deformation model with a high number of parameters [12, 9]. In this paper we propose to estimate the few parameters of the distortion geometric model from the mutual information in order to achieve a robust correction. This approach largely improves previous ones. Simpler similarity measures, indeed, seem not sufficient to perfectly take into account the complex dependencies embedded in equation 1. Moreover, the fact that a priori knowledge on the deformation main effects allows us to estimate only three paramaters per slice highly simplifies the optimization process.

This paper proposes a second improvement of the standard calculation of the diffusion tensor $D$. The linearity of Eq. 1 usually leads to a least squares based regression method [2]. This approach, however, is not robust to the various kinds of noises that can be observed in diffusion-weighted data 5. Non Gaussian noise can stem for instance from physiological motions (brain beat), subject motions or residual distortions. While careful acquisition schemes including cardiac gating and navigator echo may reduce some of these problems, some weaknesses of the tensor diffusion model lead to other regression problems: each voxel includes several water compartments endowed with different diffusion processes that are mixed up in the data [7]. Hence, in order to overcome the influence of outliers on the tensor estimation, we propose the use of a standard robust M-estimator [16. A comparison of the behaviour of both regression methods in the presence of various levels of corrupted data prove the interest of the robust approach.

\section{Distortion Correction}

In the following, echo-planar diffusion-weighted images were acquired in the axial plane. Blocks of eight contiguous slices were acquired each $2.8 \mathrm{~mm}$ thick. Seven blocks were acquired covering the entire brain corresponding to 56 slice locations. For each slice location 31 images were acquired; a T2-weighted image 


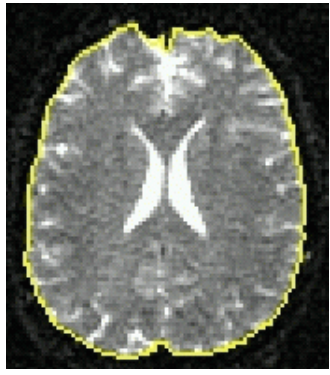

no gradient

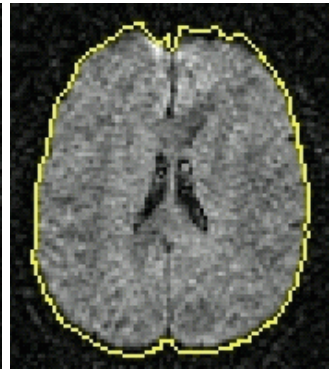

$13 \mathrm{mT} / \mathrm{m}(-1,1,0)$

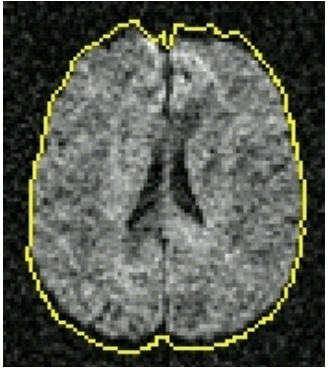

$22 \mathrm{mT} / \mathrm{m}(-1,1,0)$

Fig. 1. Example of eddy-current related distortions ( $8 \mathrm{~mm}$ in the worst case)
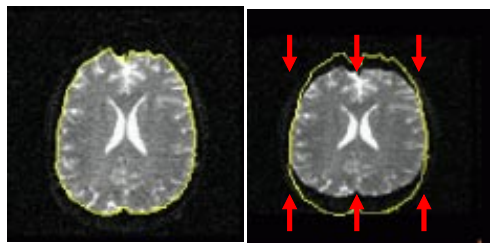

stretch

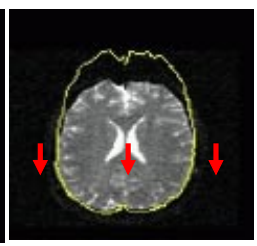

translation

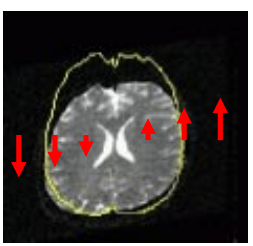

shear

Fig. 2. The simple geometric model of eddy-current related distortions

with no diffusion sensitization followed by 5 diffusion sensitized sets (b values linearly incremented to a maximum value of $1000 \mathrm{~s} / \mathrm{mm}^{2}$ ) in each of 6 noncolinear directions. In order to improve the signal to noise ratio this was repeated 4 times, providing 124 images per slice location. The image resolution was 128 $\mathrm{x} 128$, field of view $24 \mathrm{~cm} \times 24 \mathrm{~cm}, T E=84.4 \mathrm{~ms}, T R=2.5 \mathrm{~s}$

For each slice, all acquisitions are aligned with the first image of the series. For convenience we use the notation that the image is in the XY plane, and the phase-encoding direction lies along Y. Simple considerations about MR physics lead to the following distortion model [8]:

- A residual gradient in the slice-encoding direction $\mathrm{Z}$ produces uniform translation along Y;

- A residual gradient in the frequency-encoding direction $\mathrm{X}$ produces a shear parallel to $\mathrm{Y}$ (a translation linearly related to $\mathrm{X}$ );

- A residual gradient in the phase-encoding direction $\mathrm{Y}$ produces a uniform scaling in $\mathrm{Y}$ direction.

Hence, the geometric model (see Fig. 2) can be written for each column $X$ as:

$$
Y^{\prime}=S Y+T_{0}+T_{1} X
$$

An additional global multiplicative correction by $\frac{1}{S}$ has to be applied to the slice intensities, which is done after estimation of $\left(S, T_{0}, T_{1}\right)$.

To take into account the complex dependencies between the target image, which is a standard T2-weighted image, and the diffusion-weighted images, the 


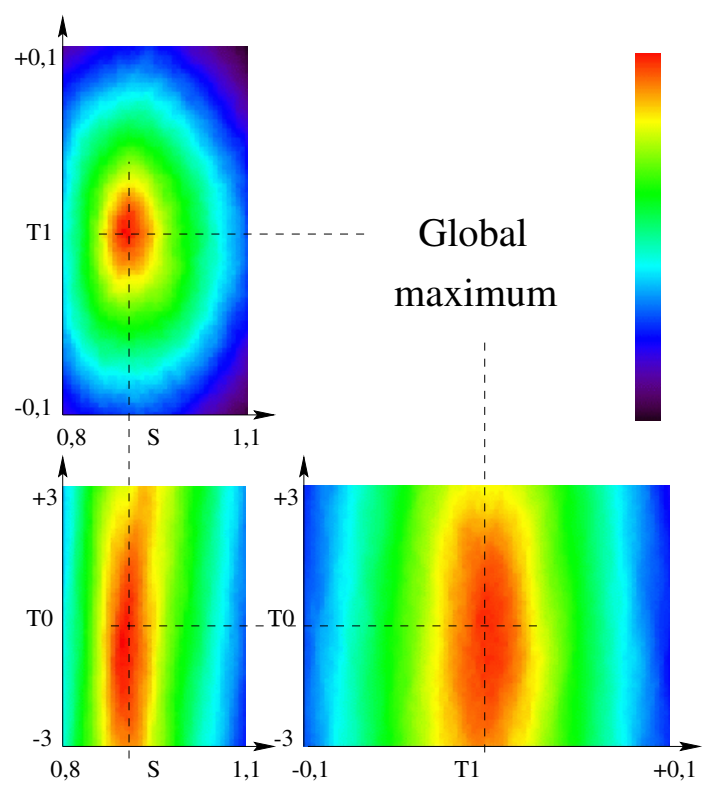

Fig. 3. Orthogonal slices of the mutual information crossing at the global maximum.

similarity measure used to estimate the optimal $\left(S, T_{0}, T_{1}\right)$ is the mutual information (MI) [10, 14. Since the two images to be aligned are $128 \times 128$ slices to be compared to the usual 3D situation, a Parzen window is used to get a robust estimation of the joint intensity distribution. This Parzen window is a truncated Gaussian kernel sufficient to smooth the joint histogram. This approach turned out to be crucial to prevent the maximization algorithm to be trapped in MI local maxima. Hence, estimation of $\operatorname{MI}\left(S, T_{0}, T_{1}\right)$ consists of a linear resampling of the image to be aligned according to Eq. 2. followed by the application of the Parzen window to the joint histogram. Then MI can be computed from:

$$
\operatorname{MI}\left(S, T_{0}, T_{1}\right)=\sum_{i_{t}=0}^{M-1} \sum_{i_{a}=0}^{M-1} p\left(i_{t}, i_{a}\right) \log \frac{p\left(i_{t}, i_{a}\right)}{p\left(i_{t}\right) p\left(i_{a}\right)}
$$

where $M$ is the sampling of the joint probability distribution (in practice $M=$ $64), i_{t}$ the intensity in the target image and $i_{a}$ the intensity in the image to be aligned. The marginal probabilities $p\left(i_{t}\right)$ and $p\left(i_{a}\right)$ are computed by row and column summation.

Since 123 realignments have to be performed for each slice of the volume, a fast optimization scheme is required. Fortunately, the use of a Parzen window leads to a rather smooth MI landscape around the global maximum (see Fig. 31). In some cases, several maxima have been observed near the global one. In such situations, however, we could not claim that the global maximum was a better solution than the surrounding maxima. Hence, Powell algorithm has been used 
in the following to maximize MI [19]. For each new image, the initial position is $(1,0,0)$, namely the no distortion situation.

\section{Correlation ratio}
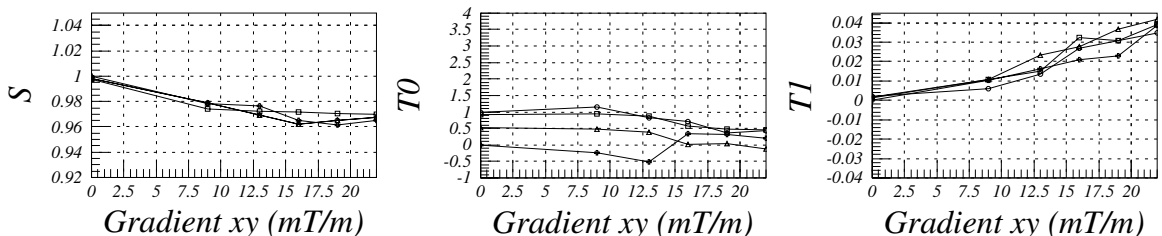

\section{Mutual information}
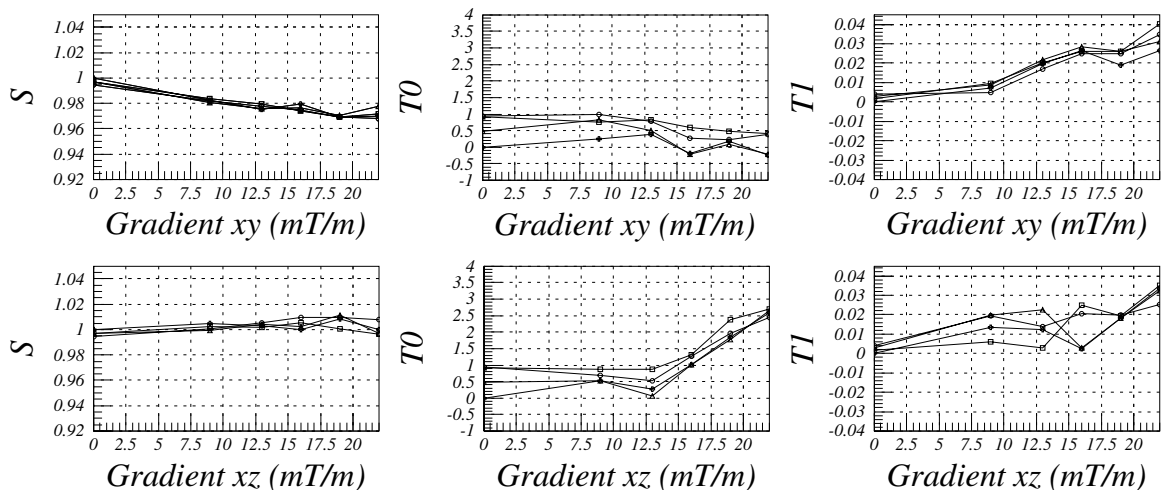

Gradient xy $(\mathrm{mT} / \mathrm{m})$
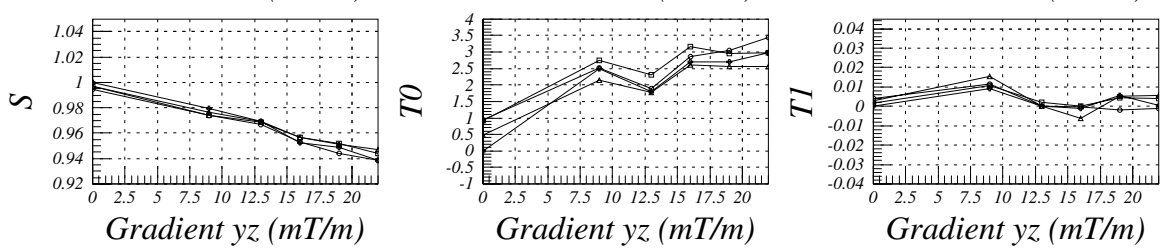

Fig. 4. Comparison between mutual information and correlation ratio for one gradient direction: $(1,1,0)$. Reproducibility of the correction process across the four repetitions in three gradient directions, $(1,1,0),(1,0,1)$ and $(0,1,1)$, with six different strengths.

Thanks to the four repetitions embedded in our acquisition process, the accuracy and the robustness of the correction process can be evaluated. A first experiment consists of comparing the results obtained using mutual information with the results obtained using another similarity measure: the correlation ratio 20. While both methods have given similar results, the variability across the four repetitions was higher for the correlation ratio (see Fig. 4). Hence, mutual information has been chosen as more adapted to diffusion-weighted images. 


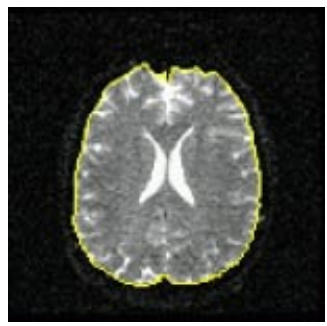

target

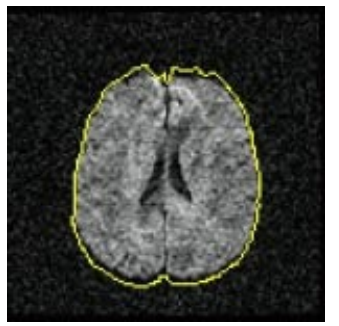

raw image

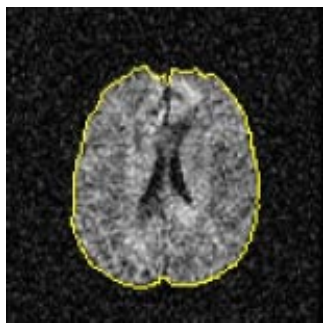

correction

Fig. 5. Example of distortion correction

In general, the correction was reproducible across the four repetitions (see Fig. 4). The largest variability was observed for the translation parameter $T_{0}$, which can be understood from the shape of MI landscape (see Fig. 3). MI isophotes, indeed, are rather cylindrical with a T0 oriented axis along which some local maxima can be observed. An interesting result is the fact that the highest variability is obtained for the three repetitions of the pure T2-weighted target (no sensitizing gradient) for which $S=1, T_{1}=0$ but $T_{0} \neq 0$. This observation tends to prove that eddy currents have long term trends that corrupt several consecutive acquisitions. Finally, the estimated distortions fit well with the physical interpretation mentioned above: The $\mathbf{x y}$ and $\mathbf{y z}$ gradients induce a scaling, the $\mathbf{x z}$ and $y \mathbf{z}$ gradients induce a global translation, and the $\mathbf{x y}$ and $\mathbf{x z}$ gradients induce a shearing.

\section{Robust Tensor Estimation}

Estimation of the diffusion tensor is done from linear equation 1. While tradition and ease of computation have made the least squares method the popular approach for this regression analysis [2], this method becomes unreliable if outliers are present in the data. Robust regression methods can be used in such situations 16. The M-estimators are the more popular robust methods. These estimators minimize the sum of a symmetric, positive-definite function $\rho\left(\epsilon_{i}\right)$ of the residuals $\epsilon_{i}$, with a unique minimum at $\epsilon_{i}=0$. A residual is defined as the difference between the data point and the fitted value. For the least squares method $\rho\left(\epsilon_{i}\right)=\epsilon_{i}^{2}$. Several $\rho$ functions have been proposed which reduce the influence of large residual values on the estimated fit. We have chosen one of the most popular ones, the Geman-McLure estimator $\rho\left(\epsilon_{i}\right)=\frac{\epsilon_{i}^{2}}{\epsilon_{i}^{2}+C^{2}}$, where $C=1.48$ median $_{i}\left\{\left\|\epsilon_{i}\right\|\right\}$. The M-estimate of the diffusion tensor is obtained by converting the minimization into an iterated weighted least squares problem. The initial guess is the solution of the standard least squares.

In order to compare the behaviour of both M-estimators, raw data have been corrupted with various levels of outliers (of course some actual outliers are also present in these data). For a given experiment, a percentage $P$ of the 124 images is modified. For such images, an additional error $e$ is added to each voxel. This 

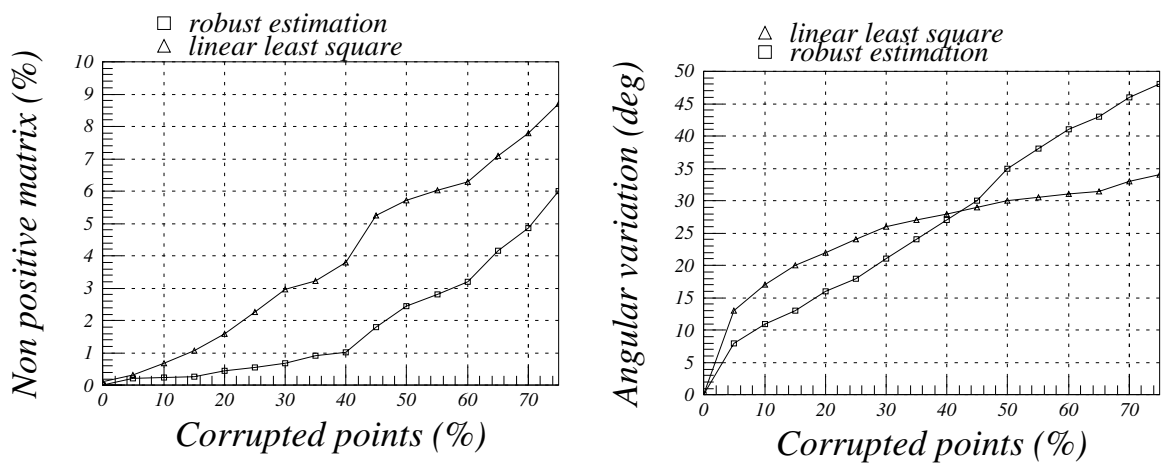

Fig. 6. Influence of outliers on the number of non positive matrices and on the direction of highest diffusion.

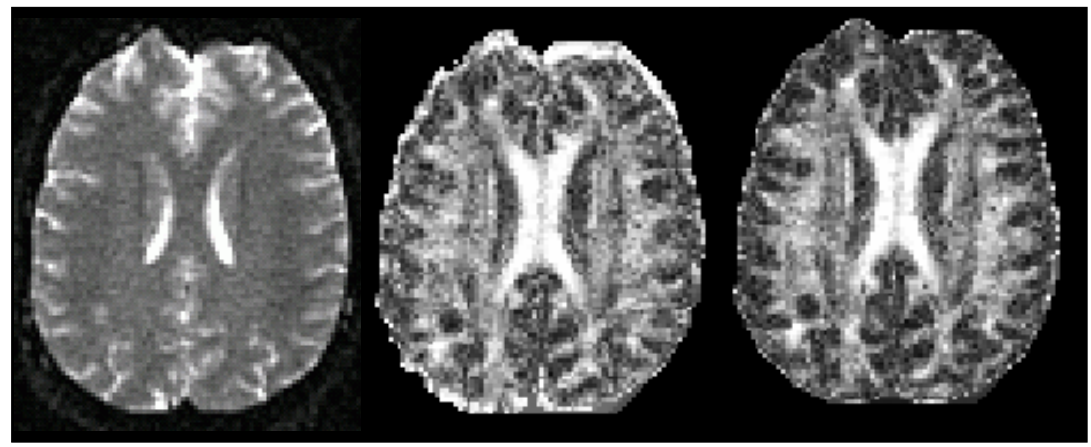

Fig. 7. Left: raw T2-weighted image. Right: tensor fractional anisotropy [17] without and with distortion correction and robust regressor. Fractional anisotropy is a simple ratio which measures the variability between the diffusion tensor eigenvalues.

error is sampled from a Gaussian distribution whose mean is the mean intensity inside the brain, and whose standard deviation is a tenth of the mean. Two measures allow us to assess the effect of these outliers. The first one is the number of non positive estimated tensors, which have no physical interpretation. Such situations may occur because no positivity constraint is embedded in the fitting process. The second measure is the mean angular variation between the direction of highest diffusion with and without outliers. This direction corresponds to the tensor eigenvector associated with the largest eigenvalue. The evolution of these measures relative to the percentage of outliers $P$ is proposed in Fig. 6. The superiority of the Geman-McLure estimator is straightforward. 


\section{Conclusion}

This paper has presented a robust procedure to estimate the diffusion tensor from a sequence of diffusion-weighted images. Further work, however, could still improve this procedure. For instance, a fitting method including a positivity constraint on the tensor eigenvalues should be designed. Furthermore, the issue of distortion correction in the presence of subject motions remains completely open, like in the case of functional MRI. Nevertheless, our new procedure already highly improves the quality of the diffusion map which is illustrated by anisotropy images in Fig. 7

\section{References}

[1] A. L. Alexander, J. S. Tsuruda, and D. L. Parker. Elimination of eddy current artefacts in diffusion-weighted echo-planar images: the use of bipolar gradient. Magn. Reson. Med., 38:1016-1021, 1997.

[2] P.J. Basser, J. Mattiello, and D. LeBihan. Estimation of the effective self-diffusiontensor from the NMR spin echo. J. of Magn. Reson., Series B, 103:247-254, 1994.

[3] P.J. Basser, J. Mattiello, and D. LeBihan. Mr diffusion tensor spectroscopy and imaging. Biophysical Journal, 66:259-267, 1994.

[4] M. E. Bastin. Correction of eddy current-induced artefacts in diffusion tensor imaging using iterative cross-correlation. Magn. Reson. Imag., 17:1011-1024, 1999.

[5] M.E. Bastin, P.A. Armitage, and I. Marshall. A theoretical study of the effect of experimental noise on the measurement of anisotropy in diffusion imaging. Magnetic Resonance Imaging, 16(7):773-785, 1998.

[6] F. Calamante, D.A. Porter, D.G. Gadian, and A. Connelly. Correction for eddy current induced B0 shifts in diffusion-weighted echo-planar imaging. Magnetic Resonance in Medicine, 41:95-102, 1999.

[7] C. A. Clark and D. LeBihan. Water diffusion compartmentation and anisotropy at high b values in the human brain. Magn. Reson. Med., 44:852-859, 2000.

[8] J.C. Haselgrove and J.R. Moore. Correction for distorsion of echo-planar images used to calculate the apparent diffusion coefficient. Magn. Reson. Med., 36, 1996.

[9] P. Hellier and C. Barillot. Multimodal non-rigid warping for correction of distortions in functional MRI. In MICCAI, LNCS-1935, pages 512-520, 2000.

[10] W. Wells III, P. Viola, H. Atsumi, S. Nakajima, and R. Kikinis. Multi-modal volume registration by maximization of mutual information. MIA, 1:35-51, 1996.

[11] P. Jezzard, A. Barnett, and C Pierpaoli. Characterization and correction for eddy current artefacts in echo planar diffusion imaging. MRM, 39:801-812, 1998.

[12] J. Kybic, P. Thévenaz, A. Nirkko, and M. Unser. Unwarping of EPI images. IEEE Trans. Medical Imaging, 19(2):80-93, 2000.

[13] D. Le Bihan, J.-F. Mangin, C. Poupon et al. Diffusion tensor imaging: concepts and applications. Journal of Magnetic Resonance Imaging, 13:534-546, 2001.

[14] F. Maes, A. Collignon, D. Vandermeulen, G. Marchal, and P. Suetens. Multimodality image registration by maximisation of mutual information. IEEE Trans. Medical Imaging, 16(2):187-198, 1997.

[15] J. Mattiello, P. J. basser, and D. LeBihan. The b matrix in diffusion tensor echoplanar imaging. Magn. Reson. Med., 37:292-300, 1997.

[16] P. Meer, D. Mintz, and A. Rosenfeld. Robust regression methods for computer vision: a review. International Journal of Computer Vision, 6(1):59-70, 1991. 
[17] C. Pierpaoli and P. J. Basser. Toward a quantitative assessment of diffusion anisotropy. Magn. Reson. Med., 36:893-906, 1996.

[18] C. Poupon, J.-F. Mangin, C. A. Clark, V. Frouin, D. LeBihan, and I. Bloch. Towards inference of the human brain connectivity from MR diffusion tensor data. Medical Image Analysis, 5:1-15, 2001.

[19] M. Powell. An efficient method for finding the minimum of a function of several variables without calculating derivatives. The Computer Journal, 7:155-162, 1964.

[20] A. Roche, G. Malandain, X. Pennec, and N. Ayache. The correlation ratio as a new similarity measure for multimodal image registration. In MICCAI'98, MIT, USA, LNCS-1496, Springer Verlag, pages 1115-1124, 1998.

[21] E.O. Stejskal and J.E. Tanner. Spin diffusion measurements: spin echoes in the presence of a time-dependent field gradient. Journal of Chemical Physics, 42(1):288-292, 1965. 